\title{
Patients with rectal cancer having neoadjuvant chemoradiation do not have increased complications of ileostomy closure
}

\author{
S R E Wijesuriya ${ }^{1}$, J Hewavisenthi², K I Deen ${ }^{1}$
}

(Index words: radiotherapy, rectal cancer, ileal damage)

\begin{abstract}
Objectives It is conceivable that reversal of an ileostomy after low anterior resection following neoadjuvant therapy (NAT) may involve anastomosis of small bowel exposed to irradiation. The aim was to evaluate peri-operative complications of ileostomy closure and to compare the histology of ileal mucosa in excised stomas in patients who received NAT with those without NAT.

Methods Twenty patients who underwent rectal excision following NAT for cancer, were compared with 20 control patients who underwent rectal excision without NAT. All patients received a diverting loop ileostomy which was subsequently reversed with excision of the ileostomy. The clinical outcome and histopathological features after reversal were evaluated.
\end{abstract}

Results There was no significant difference with regard to peri-operative complications such as post-operative deaths related to ileostomy closure, anastomotic leakage, retraction of stoma or small bowel fistulae. Resection margins revealed no significant difference in crypt distortion, depletion of mucin, acute inflammation, chronic inflammation and infiltration of eosinophils following NAT compared with controls.

Conclusions Neoadjuvant therapy for rectal cancer does not result in higher morbidity following closure of diverting loop ileostomy or result in significant inflammatory changes in the ileum. Therefore ileostomy closure is as safe in those with preoperative radiotherapy as in those without neoadjuvant therapy.

Ceylon Medical Journal 2010; 55: 115-7.

Departments of ${ }^{1}$ Surgery and ${ }^{2}$ Pathology, Faculty of Medicine, University of Kelaniya, Sri Lanka.

Correspondence: RW, e-mail: <ruwanwije@hotmail.com>.

Competing interests: none declared.

Vol. 55, No. 4, December 2010 


\section{Introduction}

Preoperative pelvic irradiation with or without chemotherapy is a recognised mode of adjuvant therapy in rectal cancer and is the preferred option in selected patients [1]. In addition to down-staging of the tumour and lower local recurrence rates, complications of radiation therapy are less when used preoperatively [2].

Gastrointestinal complications which may occur as a result of exposure of ileum, sigmoid colon and rectum to irradiation are the most distressing and common. Nausea, vomiting and diarrhoea usually occur during the initial phase of treatment and may continue for a variable period thereafter. However, complications such as intestinal obstruction, perforation and enterocutaneous fistulae have been known to occur at a later stage [3].

A diverting loop ileostomy is done after low and extended low anterior resection for rectal cancer to mitigate consequences of anastomotic leakage that have been reported to be as high as $15-20 \%$ for low rectal cancer $[4,5]$. Construction of a loop ileostomy for diversion is preferred by many surgeons because of low morbidity and improved quality of life in patients with ileostomy $[5,6]$. In patients who have been subjected to neoadjuvant therapy for rectal cancer, it is conceivable that terminal ileum may have been exposed to irradiation. Consequently, anastomotic leakage after reversal of the loop ileostomy, is an added risk. We hypothesised that closure of loop ileostomy after low anterior resection following neoadjuvant therapy (NAT) may be risky because of irradiation induced ileitis. The aim of our study was to compare perioperative complications in relation to reversal of a loop ileostomy and the histology of resected ileal mucosa in patients with rectal cancer having low anterior resection and loop ileostomy construction after neoadjuvant chemoradiation with those having ileostomy reversal following low anterior resection and loop ileostomy without neoadjuvant chemoradiation.

\section{Methods}

Forty patients who received loop ileostomy as a result of colo-anal anastomosis following excision of the rectum were recruited to this study from a surgical clinic at North Colombo Teaching Hospital Ragama, Sri Lanka. Twenty of them ( 11 males, 9 females, median age -57 years, range -26 to 72 years) received a loop ileostomy as a result of low anterior resection for rectal cancer, stage T3 or T4 who received high dose, long course neoadjuvant therapy and 20 patients $(7$ males, 13 females, median age -40 years, range -22 to 70 years) with coloanal anastomosis and loop ileostomy without preoperative pelvic irradiation.
Neoadjuvant therapy consisted of a total radiation dose of $4500 \mathrm{cGY}$ in 25 fractions over 5 weeks. External beam irradiation was delivered via two portals and the field of irradiation included the anorectum including mesorectum and pelvic lymph nodes below the fifth lumbar vertebra [5]. Fluoro-uracil was infused intravenously $\left(375 \mathrm{mg} / \mathrm{m}^{2} /\right.$ day $)$ during the first and last week of irradiation. Patients underwent low anterior resection 4-6 weeks later. A diverting loop ileostomy was constructed at the time of surgery and was reversed later once anastomotic healing was confirmed. Closure of loop ileostomy involved excision of the stoma and end to end anastomosis in a single layer of interrupted polyglactin 910 sutures.

Histopathologic evaluation of excised stomas was performed after fixation, using $10 \%$ formaline saline. Paraffin sections of resection margins were stained with hematoxyline and eosin. The following were sought: crypt distortion (CD), depletion of mucin (DM), acute inflammation (AI) chronic inflammation (CI) and infiltration of eosinophils (E). After ileostomy closure, the following clinical complications were sought: wound infection, wound dehiscence, anastomotic dehiscence, entero-cutaneous fistulae and small bowel obstruction.

All surgical procedures were performed by a single surgeon and pathologic evaluation of specimens were performed by the same pathologist. Data are expressed as median and range. Statistical analysis was performed using SPSS version17. Significance was assigned to a $p$ value of less than 0.05 . The project was approved by the Institutional Ethics Review Committee and informed written consent was obtained from all patients.

\section{Results}

There were no deaths or anastomotic leaks consequent to loop ileostomy closure in either group. The median time to ileostomy closure was, NAT 8 weeks (range 8-52 weeks) versus, without NAT, 8 weeks (range 8-40 weeks). One patient who had NAT was noted to have a retracted ileostomy. There was no significant difference in hospital stay following closure of ileostomy in both groups \{NAT vs controls; median - 5 days (range 4-8 days) vs 5 days (4-7 days) $\}$.

Histopathologic assessment of resection margins of excised stomas revealed no significant difference in crypt distortion \{CD[NAT 13 (65\%) vs controls $9(45 \%)$, $p=0.34]\}$, depletion of mucin $\{$ DM[NAT $5(26.6 \%)$ vs controls $3(15 \%), p=0.60]\}$, acute inflammation $\{$ AI[NAT $9(45 \%)$ vs controls $3(15 \%), p=0.084]\}$, chronic inflammation \{CI[ NAT $19(95 \%)$ vs controls $18(90 \%)$, $p=1.0]\}$ and infiltration of eosinophils $\{$ E[NAT $11(55 \%)$ vs controls $5(25 \%), p=0.10]$; z-test $\}$ following NAT compared with non irradiated controls (Table 1). 
Table 1. Histopathology of excised ileostomy; with and without neoadjuvant therapy

\begin{tabular}{lccc}
\hline & $\begin{array}{c}\text { With neoadjuvant } \\
\text { therapy }(n=20)\end{array}$ & $\begin{array}{c}\text { Without neoadjuvant } \\
\text { therapy }(n=20)\end{array}$ & $p$ value \\
\hline Crypt distortion (CD) & $13(65 \%)$ & $9(45 \%)$ & 0.34 \\
Depletion of mucin(DM) & $5(26 \%)$ & $3(15 \%)$ & 0.60 \\
Acute inflammation (AI) & $9(45 \%)$ & $3(15 \%)$ & 0.08 \\
Chronic inflammation (CI) & $19(95 \%)$ & $18(90 \%)$ & 1.0 \\
Infiltration of eosinophils (E) & $11(55 \%)$ & $5(25 \%)$ & 0.10 \\
\hline
\end{tabular}

\section{Discussion}

The impact of pelvic irradiation on normal tissue is determined by the dose of radiation, fractionation and radiosensitivity of the tissue. Exposure of bowel to irradiation results in many histopathologic changes. Microscopic features of small bowel following irradiation have been described in rat models [7]. Our data have shown that the histology of ileum after a median of 16 weeks following neoadjuvant therapy was not significantly different from non-irradiated ileum. The presence of inflammatory changes in ileum of both groups in similar proportions is most likely due to ileal exteriorisation and not exposure to irradiation. Thus our study confirms that there are either minimal or no effects of irradiation of small bowel during preoperative chemoradiation for rectal cancer. In addition the presence of any inflammatory changes which are attributable to exteriorisation appears to have no bearing on the outcome of ileostomy closure as perioperative complications following closure of ileostomy were minimal in both groups of patients. Patients who receive postoperative pelvic irradiation are more likely to sustain small bowel injury compared with preoperative irradiation, as small bowel adhesions are likely to be in the pelvis after rectal excision [8].

We conclude that neoadjuvant therapy for rectal cancer does not result in either significant inflammatory changes in small bowel or ileostomy closure related complications. Therefore ileostomy closure is as safe in those with preoperative radiotherapy as in those without neoadjuvant therapy.

\section{References}

1. Bosset JF, Pelissier EP, Maniion G, et al. Plea for a preoperative adjuvant approach in the management of rectal cancer. International Journal of Radiation Oncology Biology Physics 1994; 29: 205-8.

2. Ooi BS, Tjandra JJ, Green MD. Morbidities of adjuvant chemotherapy and radiotherapy for resectable rectal cancer: an overview. Diseases of the Colon and Rectum 1999; 42: 403-18.

3. Meissner K. Late radiogenic small bowel damage: guidelines for the general surgeon. Digestive Surgery 1999; 16: $169-74$.

4. Gastinger I, Marusch F, Steinert R, et al. Working Group 'Colon/Rectum Carcinoma'. Protective defunctioning stoma in low anterior resection for rectal carcinoma. British Journal of Surgery 2005; 92: 1137-42.

5. Platell C, Barwood N, Makin G. Clinical utility of a defunctioning loop ileostomy. Australia New Zealand Journal of Surgery 2005; 75: 147-51.

6. Silva MA, Ratnayake G, Deen KI. Quality of life of stoma patients: temporary ileostomy versus colostomy. World Journal of Surgery 2003; 27: 421-4.

7. Becciolini A, Fabbrica D, Cremonini D, Balzi M. Quantitative changes in the goblet cells of the rat small intestine after irradiation. Acta Radiologica Oncology 1985; 24: 291-9.

8. Nuyttens JJ, Robertson JM, Yan D, Martinez A. The position and volume of the small bowel during adjuvant radiation therapy for rectal cancer. International Journal of Radiation Oncology, Biology, Physics 2001; 51: 1271-80. 